\title{
Delivery of Functional Exogenous Proteins by Plant Vesicles to Human Cells in Vitro
}

\section{Luiza Garaeva}

NRC "Kurchatov Institute" -PNPI

\section{Roman Kamyshinsky}

Kurchatov Institute: Nacional'nyj issledovatel'skij centr Kurcatovskij institut

\section{Yury Kil}

NRC "Kurchatov Institute"-PNPI

\section{Elena Varfolomeeva}

NRC "Kurchatov Institute"-PNPI

\section{Yuri Garmay}

NRC "Kurchatov Institute"-PNPI

\section{Sergey Landa}

NRC "Kurchatov Institute"-PNPI

\section{Vladimir Burdakov}

NRC "Kurchatov Institute"-PNPI

\section{Alexander Myasnikov}

NRC "Kurchatov Institute"-PNPI

\section{Alexander Kagansky}

Far Eastern Federal University School of Biomedicine: Dal'nevostochnyj federal'nyj universitet shkola biomediciny

\section{llya A. Vinnikov}

Shanghai Jiao Tong University Medical Library: Shanghai Jiao Tong University School of Medicine

\section{Elena Komarova}

Institute of Cytology Russian Academy of Sciences: FGBUN Institut citologii Rossijskoj akademii nauk

\section{Boris Margulis}

Institute of Cytology RAS: FGBUN Institut citologii Rossijskoj akademii nauk

\section{Irina Guzhova}

Institute of Cytology Russian Academy of Sciences: FGBUN Institut citologii Rossijskoj akademii nauk

\section{Andrey L. Konevega}

NRC "Kurchatov Institute"-PNPI

\section{Tatiana Shtam ( $\sim$ shtam_ta@pnpi.nrcki.ru )}

NRC "Kurchatov Institute"-PNPI https://orcid.org/0000-0003-0651-4785 
Research

Keywords: grapefruit-derived extracellular vesicles (GF-EVs), exosomes, drug delivery systems, HSP70, Cryo-EM, edible plant-derived exosome-like nanoparticles (EPDELNs)

Posted Date: September 24th, 2020

DOl: https://doi.org/10.21203/rs.3.rs-79427/v1

License: (c) (i) This work is licensed under a Creative Commons Attribution 4.0 International License.

Read Full License 


\section{Abstract}

Background: Plant-derived extracellular vesicles increasingly gain attention as promising carriers of exogenous bioactive molecules to the human cells. Due to their various edible sources they are distinctly biocompatible, biodegradable and easily available in significant amounts.

Methods: In present work, extracellular vesicles from grapefruit juice were isolated by differential centrifugation and characterized in terms of size, quantity, and morphology by nanoparticle tracking analysis, dynamic light scattering, atomic force microscopy, and cryo-electron microscopy (Cryo-EM). In Cryo-EM experiments we have visualized grapefruit vesicles with average size of $41 \pm 13 \mathrm{~nm}$, confirmed their round-shaped morphology and estimated the thickness of their lipid bilayer as $5.3 \pm 0.8 \mathrm{~nm}$.

Results: Using in vitro cell culture models, we have shown that grapefruit-derived extracellular vesicles (GF-EVs) are highly efficient carriers for the delivery of the exogenous Alexa flour 647 labelled BSA and HSP70 proteins into human colon cancer HCT-116 and DLD1 cells. Both proteins when loaded to plant vesicles were captured by human intestinal cells much more efficiently compare to their free state. Additionally, the functional activity of human recombinant HSP70 delivered by GF-EVs in the tissue culture cells has been confirmed.

Conclusions: The results clearly indicate the high potential of native plant vesicles for the safe delivery of therapeutic proteins into human cells. Here, we reported the first demonstration of effective loading of natural plant-derived extracellular nanovesicles with exogenous proteins and their successful delivery into human cells.

\section{Background}

Extracellular vesicles (EVs), including exosomes, are nanoscale membrane-enclosed particles implicated in intercellular communication to facilitate transport of proteins and genetic material [1-3]. Due to their natural properties, exosomes are capable of migrating from one cell to another, passing their contents across the cell membrane, and delivery of biologically active cargoes [4]. Since exosomes offer distinct advantages for the efficient and safe delivery of biomolecules, the interest in using them as carriers has exploded in recent years [5, 6]. EVs have been reported to be used for the delivery of siRNAs [7], miRNAs [8], proteins [9], small molecule drugs [10], and CRISPR/Cas9 molecular toolkits [11] on the road to the treatment of various diseases (reviewed in [12]).

However, there is a number of limitations associated with the use of human exosomes as drug delivery vehicles. One of the major challenges in developing exosome-based formulations is the concern whether (and if so then, how?) the sufficient number of human exosomes can be generated in vitro or isolated from biological fluids. Indeed, the exosome yield per unit of starting material will impact the final production cost as well as clinical applications. In this respect, the choice of alternative sources of nanovesicles is crucial. The use of vesicles innately formed in plant cells as delivery agents could potentially solve problems associated with the existing nano-delivery systems. Firstly, edible plant-derived 
exosome-like nanoparticles (EPDELNs) are natural particles, which allows their isolation from affordable edible plants in significant quantities and eliminates any possible concerns with regards to their toxicity

$[13,14]$. Secondly, plant vesicles are natural carriers of different biomolecules, including small non-coding RNAs [15] and, therefore, are designed to maintain stability of their molecular cargo while facilitating cellto-cell communication $[16,17]$. Such properties of plant-derived extracellular vesicles (PEVs) suggest the possibility of their applications involving the ectopic cargo. Although the lipid composition of plant vesicles is different from that of mammalian exosomes [18], which could impede the effective loading of PEVs by any exogenous bioactive molecules, as well as their release in the destination cells, the efficiency of their internalization by animal cells have been confirmed in experiments using PKH26 labelled particles or in delivery of methotrexate [19]. In general, PEVs are much less studied than the vesicles secreted by mammalian cells, and their structural and functional features need to be elucidated in detail.

In the present study we have isolated extracellular vesicles from grapefruit juice by differential centrifugation and characterized them in size, quantity, and morphology by Nanoparticle Tracking Analysis (NTA), Dynamic Light Scattering (DLS), Atomic Force Microscopy (AFM), and Cryo-Electron Microscopy (cryo-EM). Finally, edible nanovesicles derived from grapefruit have been investigated for their potential use for the functional protein delivery into the intestinal human cells in vitro. Using tissue culture models, we have shown that GF-EVs are highly efficient in the delivery of exogenous recombinant human heat shock protein 70 (HSP70) into various human colon cancer cells.

\section{Materials And Methods}

\section{Reagents}

The following reagents were used in the study: Alexa Fluor 647 Protein Labeling Kit (Invitrogen, USA), Bradford reagent (BioRad, USA), Clarity Western ECL Blotting Substrate (Bio-Rad, USA), mouse monoclonal antibody to human HSP70 (Abcam, ab2787). Recombinant human HSP70 protein was isolated and chaperone activity of the protein was established by the specially designed assays [20]. All other reagents used in the study were obtained from Sigma-Aldrich (USA).

\section{Isolation and purification of vesicles from fruit parts of Citrus x paradisi (grapefruits)}

Grapefruits were purchased from a local market and washed three times with distilled water. The juice was extracted using a household citrus juicer. The collected volume $(450 \mathrm{~mL})$ of initial juice was sequentially centrifuged at $1,200 \times \mathrm{g}$ for $20 \mathrm{~min}, 3$ times at $3,000 \times \mathrm{g}$ for $20 \mathrm{~min}, 10,000 \times \mathrm{g}$ for $60 \mathrm{~min}$, and $15,000 \times \mathrm{g}$ for $60 \mathrm{~min}$ to remove large particles and cellular debris. The supernatant was centrifuged again at $10,000 \times \mathrm{g}$ overnight. The sequential ultracentrifugation method was further applied; it included centrifugation of the final supernatant on a Beckman Coulter centrifuge (Ti45 rotor, Beckman Coulter, USA), at $150,000 \mathrm{~g}$ for $2 \mathrm{~h}$. After first centrifugation, the supernatant was removed, and the pellet was 
carefully resuspended in $1 \mathrm{~mL}$ of phosphate-buffered saline (PBS) using gentle swaying overnight, then the volume was adjusted to $5 \mathrm{~mL}$ and re-centrifuged at 150,000 $\mathrm{g}$ for $2 \mathrm{~h}$ (SW 55Ti rotor, Beckman Coulter, USA). The resulting pellet was re-suspended with gentle shaking in $500 \mu \mathrm{L}$ of PBS for at least $1 \mathrm{~h}$ at $4^{\circ} \mathrm{C}$. Final samples of grapefruit-derived nanovesicles were aliquoted, rapidly frozen in liquid nitrogen and stored at $-80^{\circ} \mathrm{C}$ until the analysis.

\section{Nanoparticle Tracking Analysis (NTA)}

The size of GF-EVs and their concentration in suspensions were determined by NTA using the NanoSight LM10 (Malvern Instruments) analyzer, equipped with a blue laser ( $45 \mathrm{~mW}$ at $488 \mathrm{~nm}$ ) and a C11440-5B camera (Hamamatsu Photonics K.K., Japan). Recording and data analysis were performed using the NTA software 2.3. The following parameters were evaluated during the analysis of recordings monitored for $60 \mathrm{~s}$ : the average hydrodynamic diameter, the mode of distribution, the standard deviation, and the concentration of vesicles in the suspension.

\section{Dynamic light scattering (DLS)}

The distribution of GF-EVs in size was evaluated by the method of DLS using a PLSS laser correlation spectrometer (INTOX MED LLC, Russia) as described earlier [21]. Measurements were carried out at $+25^{\circ} \mathrm{C}$. For each sample, the particle size distribution curves were plotted according to the results of three measurements.

\section{Atomic force microscopy (AFM)}

Detection of GF-EVs was carried out by AFM. Briefly, sample of GF-EV suspension in PBS was diluted 50fold with deionized water, and $0.5 \mu \mathrm{L}$ aliquots were deposited onto freshly cleaved mica. After drying completely at room temperature, the mica surface was flooded with the excess of deionized water to dissolve salt. The remaining water was removed with compressed air after a 5-minute incubation. The sample topography measurements were performed in semi-contact mode using the atomic force microscope "NT-MDT-Smena B" with a NSG03 probe (NT-MDT, Russia). The images were analyzed using "Gwyddion" software [22].

\section{Cryo-electron microscopy}

Direct visualization of the grapefruit-derived vesicles was performed by Cryo-EM as described previously for vesicles isolated from human fluids [23]. The aqueous solution of the sample was applied on glowdischarged lacey carbon EM grid, which was then plunge-frozen into the precooled liquid ethane with Vitrobot Mark IV (ThermoFisher Scientific, USA). The samples were studied using a cryo-electron microscope Titan Krios 60-300 TEM/STEM (ThermoFisher Scientific, USA), equipped with TEM direct 
electron detector Falcon II (ThermoFisher Scientific, USA) and Cs image corrector (CEOS, Germany) at accelerating voltage of $300 \mathrm{kV}$. To minimize radiation damage during image acquisition low-dose mode in EPU software (ThermoFisher Scientific, USA) was used.

\section{Loading of grapefruit-derived vesicles with proteins}

A combination of passive and active cargo loading was used. Recombinant human HSP70 protein or BSA at a final concentration of $0.1 \mathrm{mg} / \mathrm{mL}$ was mixed with suspension of GF-EVs at a final concentration of $10^{13}$ particles $/ \mathrm{mL}$ and incubated overnight at $4^{\circ} \mathrm{C}$. Then, the mixture was sonicated at a frequency of $35 \mathrm{kHz}$ for $15 \mathrm{~min}$ at RT by the Bandelin SONOREX SUPER ultrasonic bath (Bandelin Electronic GmbH \& Co. $\mathrm{KG}$ ) at room temperature, and incubated for additional $90 \mathrm{~min}$ at $4^{\circ} \mathrm{C}$. To remove the excess of free proteins, the vesicles were purified using ultrafiltration through a 100-kDa filter (Amicon, Millipore) ten times with washing by PBS. The first and tenth eluates in filtration procedure (F1 and F10) were used as controls in further experiments. The obtained suspension of "protein-loaded" grapefruit vesicles was adjusted to the starting volume of the initial suspension of GF-EVs with PBS. The final concentration of loaded GF-EVs was established by NTA. The total protein amount of GF-EVs, F1, F10, and GF-EVs loaded with HSP70 was determined using Bradford reagent (BioRad, USA). For some experiments Alexa Fluor 647 labelled variants of HSP70 and BSA (HSP70-AF647 and BSA-AF647) were used for loading to GF-EVs in a similar manner.

\section{Western blotting}

The presence of HSP70 protein in the samples of loaded GF-EVs was determined by western blotting. The purified samples of GF-EVs loaded with recombinant HSP70 were incubated at $4{ }^{\circ} \mathrm{C}$ for 30 minutes with $20 \mu \mathrm{L}$ of lysis buffer (7M urea, $2 \mathrm{M}$ thiourea, $4 \%$ CHAPS, $5 \mathrm{mM} \mathrm{PMSF}, 1 \% \mathrm{DTT}$ ). The same number of vesicles isolated from grapefruit (without loading procedure) was analyzed in parallel. The protein samples were diluted in Laemmli buffer (BioRad, USA), subjected to $10 \%$ SDS-PAGE containing $0.1 \%$ SDS, and transferred to the PVDF membrane (Thermo Scientific) using the Trans-Blot Turbo Transfer System (BioRad, USA). Immunoblotting was performed according to the Blue Dry Western protocol [24]. Mouse monoclonal antibodies to HSP70 (Abcam, ab2787) were used as primary antibodies at 1:200 dilution. Horseradish peroxidase-conjugated goat antibodies against mouse immunoglobulins were used as secondary antibodies at 1:10,000 dilution. Chemiluminescent detection of the protein bands was performed with Clarity Western ECL Blotting Substrate (Bio-Rad, USA) and Thermo Scientific CL-XPosure Films (Thermo Fisher Scientific, USA). Recombinant human HSP70 protein (10 $\mu \mathrm{g})$ was used as a positive immunodetection control.

\section{GF-EVs mediated delivery of mammalian proteins into human cancer tissue culture}


HCT116 and DLD1 human colon cancer cell lines were obtained from the Cell Culture Collection, Institute of Cytology of the Russian Academy of Science. Cells were cultured in DMEM-F12 (BioLot, Russia) containing $10 \% \mathrm{FBS}$ (Hyclone) at $37^{\circ} \mathrm{C}, 5 \% \mathrm{CO}_{2}$. Cultured DLD1 cells were stably transfected with pcDNA3 (DLD1-scr) or pcDNA3-HSP70 (DLD1-hsp70) plasmids as previously described [25]. The resulting cells expressed GFP alone or $h s p 70$ shRNA and GFP, respectively.

For protein delivery assays, HCT116, DLD1, DLD1- scror DLD1-hsp70 cells were seeded on 12-well plates at a density of $1 \times 10^{5}$ cells/well. In order to deliver the exogenous proteins, purified samples of proteinloaded GF-EVs were co-cultured with the recipient cells. The number of loaded vesicles was determined by NTA and the equivalent number of vesicles was added ( $10^{6}$ vesicles/cell). After $1 \mathrm{~h}$ of incubation, the cells transfected via GF-EVs with fluorescently labeled HSP70 or BSA were trypsinized and collected, then washed three times with PBS and analyzed by Confocal microscopy (LEICA TCS SP5X) and Flow cytometry (Beckman Coulter).

To determine the functionality of the HSP70 protein delivered by GF-EVs, the proliferative activity of recipient cells in the presence of etoposide was analyzed. DLD1 scr, DLD1- $h s p 70$ cells were seeded in 16well $x$ CELLigence E-Plate at a density of $1 \times 10^{4}$ cells/well and cultured overnight. Then grapefruit vesicles loaded with HSP70 and control vesicles (not loaded with HSP70) at the same concentration of $10^{6}$ particles per cell were added. Control samples, including only HSP70 protein $(0,05 \mathrm{mg} / \mathrm{mL})$, as well as F1 and F10 filtrates, obtained by ultrafiltration of loaded particles through 100-kDa filter, were cocultivated in parallel. After 4 hours, etoposide at a concentration of $20 \mu \mathrm{M}$ was added. A comparative analysis of the proliferative activity of cells in the presence of etoposide was performed in real time using instrument xCELLigence RTCA DP System (ACEA Biosciences, Inc, USA). All samples were duplicated within the same experiment.

\section{Results}

\section{Characterization of grapefruit-derived nanovesicles}

Vesicles were isolated from $400 \mathrm{~mL}$ of grapefruit juice by sequential ultracentrifugation according to the purification protocol for edible plant-derived exosome-like nanoparticle described earlier with some modifications [15]. In order to characterize grapefruit-derived extracellular vesicles, their size distribution and concentration were measured by NTA. In the purified samples of GF-EVs the following parameters were measured: size mode and concentration of vesicles in suspension. These parameters were determined as $52 \pm 8 \mathrm{~nm}$ and $(5.7 \pm 0.7) \times 10^{13}$ particles/mL, respectively (Fig. 1a). Since the NTA method gives a wide size distribution, we further performed the sizing of vesicles isolated from grapefruit using DLS. Three distinct peaks were detected by DLS: $28.3 \pm 9.8 \mathrm{~nm}, 79.6 \pm 6.0 \mathrm{~nm}$, and $270.0 \pm 46.2 \mathrm{~nm}$ (Fig. 1b). The majority of particles ( $90.0 \pm 3.7 \%$ contribution by mass) had a size of about $30 \mathrm{~nm}$.

Surface topology of grapefruit nanovesicles was estimated by AFM. In several samples of vesicles isolated from grapefruit, we have observed individual particles of spherical shape that corresponded to 
vesicular topology with diameters ranging from 50 to $120 \mathrm{~nm}$, and heights from 30 to $60 \mathrm{~nm}$. In addition, a number of small particles with heights of about $15 \mathrm{~nm}$ were also observed (Fig. 2).

\section{Morphological characterization of EVs by cryo-EM}

The morphology of grapefruit-derived particles was characterized using cryo-EM (Fig. 3). Since the majority of the particles had a round-shaped vesicular morphology formed by a characteristic lipid bilayer with an average thickness of $5.3 \pm 0.8 \mathrm{~nm}$ (Fig. $3 a, b, d)$ these particles were defined as grapefruit-derived extracellular vesicles (GF-EVs). Among them single vesicles containing electron dense material were visualized (Fig. 3a, d). The average size of GF-EVs was $41 \pm 13 \mathrm{~nm}$ (Fig. 3e). The small amount of larger vesicles (up to $200 \mathrm{~nm}$ ) was also observed (Fig. 3a, b, e). The presence of vesicles with altered morphology, such as double (Fig. 3b) and elliptical vesicles (Fig. 3c), was insignificant.

\section{Introduction of exogenous protein into grapefruit nanovesicles and delivery of the protein to recipient cells via GF-EVs}

First, we investigated the possibility of GF-EVs loading with exogenous cargoes using sonication. As a procedure for transfection of the plant vesicles is not well-characterized, recombinant human HSP70 protein was used for empirical optimization of the protocol. The best result was demonstrated using passive protein penetration in combination with sonication. The sonicated mixture was purified 10 times by washing and ultrafiltration through a $100-\mathrm{kDa}$ filter to eliminate the excess of free proteins. At the end of the loading procedure, all samples, including the final suspension of loaded vesicles, the first and tenth filtrates, were brought to volume equal to the starting volume of the initial suspension of GF-EVs. A schematic representation of the GF-EVs loading procedure with an exogenous HSP70 is shown in Figure 4a. The concentration of GF-EVs measured using NTA did not change significantly during the loading procedure and sample washing. As determined using the protein assay, the first filtrate (F1) contained a significant amount of free protein, while the last filtrate (F10) was actually protein free. The loading efficiency of GF-EVs with human HSP70 protein was estimated by Western blotting using the equivalent number of particles (Fig. 4b). In further experiments the first and the last wash filtrates were referred to as positive and negative controls, respectively.

Grapefruit vesicles loaded with either HSP70 or BSA labeled with Alexa Flour 647 (HSP70-AF647 and BSA-AF647) were co-cultured with the recipient human colon cancer cells HCT116 and DLD1 in order to transfect cells with the exogenous proteins. High delivery efficiency of either protein to recipient cells by GF-EVs was confirmed by flow cytometry and confocal microscopy (Fig. 5a, e, f). Moreover, fluorescence signal accumulation was observed with increasing incubation time of recipient cells and GF-EVs loaded with HSP70-AF647 (Fig. 5b, g). Colon cancer cells were also co-cultured with first (F1) and tenth (F10) filtrates as positive and negative controls, respectively. The results from flow cytometry showed that the 
fluorescence signal from labeled proteins could be detected in the cells co-cultured with the first filtrates containing the free proteins (Fig. 5c, d). The cell uptake efficiency towards free human HSP70 appeared to be significantly higher than towards BSA. Importantly, both BSA and HSP70 loaded to GF-EVs were captured by human cells much more efficiently than the free proteins (Fig. 5c, d). As expected only a faint fluorescent signal was detected in control cells incubated with tenth eluate F10, confirming that the purification procedure used was sufficient to remove free protein (not loaded to vesicles) from the samples.

Protein-loaded GF-EVs were effectively internalized by all types of investigated colon cancer cells. A layerby-layer scanning of cells using confocal microscopy visualized labeled proteins within the cytoplasm of recipient cells (Fig. 5e, f). The fluorescence signal in the recipient cells have been registered as soon as 30 min into the incubation of cells together with loaded grapefruit vesicles, and the fluorescence intensity kept increasing upon further incubation.

The accumulation of fluorescent protein delivered to the recipient cells by GF-EVs was detected in real time using confocal microscopy (Fig. 5g, Supplementary Figure S1). Thus, our results indicate the effective protein delivery into human cells by GF-EVs.

\section{Functionality of HSP70 protein delivered by GF-EVs to human colon cancer recipient cells}

Next, we tested whether the cargo protein HSP70 leaves the plant vesicles in the biologically functional state inside the recipient cells using an in vitro model, where we compared the etoposide sensitivity of recipient cells co-cultivated with either HSP70-loaded GF-EVs or free HSP70 protein. Etoposide is a chemotherapeutic agent that inhibits cell growth and induce apoptosis in a variety of tumor cells. The HSP70 chaperone prevents etoposide anti-proliferative effect [26]. To test the functionality of the HSP70 protein delivered to the cells by GF-EVs, we analyzed the proliferative activity of colon cancer DLD1-scr or DLD1-hsp70 cells in the presence of etoposide in real time (Fig. 6). The growth curves of both cell lines in the presence of intact GF-EVs (without load procedure) did not differ from the control curves, which indicates the absence of any toxic or stimulating effect of the plant particles. The addition of etoposide alone led to proliferation decrease and cell death of both lines, as expected (Fig. 6, red line). However, the addition of etoposide to cells preincubated with either HSP70 (Fig. 6, green line) or HSP70-loaded GF-EVs (Fig. 6, blue line) did not inhibit cell proliferation compared to when cells were cultured with etoposide only. Moreover, the observed protective effect was dose-dependent, correlating with the amounts of GFEVs loaded with HSP70 (Fig. 6, dark and light blue lines), and it was approximately the same for analyzed cell lines. These data demonstrate that the HSP70 protein remains fully functional when delivered to human recipient cells by GF-EVs.

\section{Discussion}


Over the last decade, extracellular vesicles, especially human exosomes, were considered promising carriers of different exogenous bioactive molecules to the human cells. Among these agents, therapeutic molecules of RNA, peptides or proteins that problematic for delivery by standard methods were of the main focus. Human EVs from various biofluids are well studied [2], and much progress has been achieved in using them as drug delivery systems $[27,28]$.

In the recent years, different strategies of exosome loading with various exogenous cargoes have been development and optimized [12, 29]. Haney et al. [9] reported the high efficiency of sonication, extrusion, or permeabilization with saponin for loading human macrophage-derived exosomes with catalase. Furthermore, intranasal administration of catalase-loaded exosome led to behavioral recovery in murine model of Parkinson's disease demonstrating that exosome can cross the blood brain barrier for brain tumor therapy [9].

However, further development of drug-loaded EVs targeted delivery technologies is required for wider therapeutic application. Moreover, it calls for the availability of large amounts of pure exosomes is necessary. The extracellular vesicles, isolated from edible plants, provided their efficiency and abundance, are considered promising for these purposes [30]. Particularly plant-derived exosome-like nanoparticles have several distinct advantages over exosomes of animal origin. They are biocompatible and biodegradable, could be isolated in large amounts, and therefore plants may become raw materials of choice to isolate safer tools for the delivery of therapeutic agents.

In the recent years, the molecular content of EVs from various plant sources, mostly edible, (fruits and vegetables, starchy roots and tubers, nuts and seeds, fresh and dried plants) have been investigated. Several studies discuss that plant EVs release is stimulated by pathogen infection and stress, while others - that plant EVs contain proteins, various RNAs, polysaccharides, and lipid signals, related to their roles in plant defense [31, 32]. Analysis of protein contents of PEVs isolated from different sources revealed several interesting proteins, which are considered as potential specific markers, such as PENETRATION1, Patellins 1-3, Clathrin heavy chain, as well as heat shock proteins [33,34]. However, plant extracellular vesicles are still barely characterized, which calls for more in-depth studies considering their potential applications.

In the present study, EVs from grapefruit juice have been characterized by their size, quantity, and morphology by commonly used in nanoparticle size and shape interpretation methods, including NTA, DLS and atomic force microscopy. As the population of isolated EVs is usually heterogeneous in size, origin and molecular constituents, different techniques applied for their characterization have some limitations [35]. Cryo-EM method based on direct imaging of single particles in their close-to-native state provides the most reliable data for extracellular vesicle size and shape determination. Indeed, cryoelectron microscopy of EVs allowed us to obtain high-quality images of grapefruit membrane-enclosed vesicles and accurately determine their size distribution. Most of the isolated GF-EVs were single, roundshaped, and in a size range from 30 to $55 \mathrm{~nm}$ (Fig. 3). To our knowledge, this is the first visualization of plant-derived vesicles with a high enough resolution to allow the estimation of their lipid bilayer 
membrane thickness as $5.3 \pm 0.8 \mathrm{~nm}$. In general, cryo-EM data are in accordance with the analysis of grapefruit-derived nanovesicles by other methods based on the single or the ensemble particle sizing approaches, NTA (Fig. 1a), DLS (Fig. 1b), and AFM (Fig. 2). Previously, several studies have reported that ultracentrifugation may cause aggregation or morphological changes of EVs [36, 37], including double and multilayer structures, which could be observed in substantial amounts in many exosome samples, prepared from human biological fluids or cell culture medium [23, 38, 39]. While grapefruit-derived nanovesicles were purified with more stringent centrifugation, cryo-EM visualization did not show any significant nanovesicle aggregation or double particle formation in the obtained suspension of GF-EVs.

In the current study, we have also tested the loading of native grapefruit vesicles with protein cargoes using sonication technique, evaluated the capture efficiency of plant vesicles by human colon cancer tissue culture cells, and demonstrated the functionality of the delivered proteins. Using in vitro cell culture models, we have shown that fluorescence labeled proteins HSP70-AF647 or BSA-AF647 loaded to GF-EVs were captured by human cells much more efficiently than the free proteins. Taken together with additional confirmation of the functional activity of human recombinant HSP70 upon delivery by grapefruit vesicles our results demonstrate that GF-EVs are highly efficient for delivering a functional exogenous protein to human cells. Grapefruit-derived nanovesicles have been studied previously in several works $[18,19,40]$. Using both in vitro cell culture models as well as mouse models, the authors have shown that nanoparticles made of grapefruit-derived lipids are highly efficient for delivering a variety of therapeutic agents, including drugs, DNA expression vectors, siRNA, and antibodies [18]. In work [40], the enhancement for homing to tumor tissues have been demonstrated for grapefruit-derived nanovectors coated with inflamatory-related receptor enriched membranes of activated leukocytes. In general, recent investigations provide a strong basis for further study and development of plant vesicle delivery systems and their use in cancer treatment and precision medicine.

\section{Conclusion}

Here, we reported the first demonstration of effective loading of natural plant-derived extracellular nanovesicles with exogenous proteins and their successful delivery into human cells. The results of our study clearly indicate the high potential of native plant vesicles for the safe delivery of therapeutic proteins into human cells.

\section{List Of Abbreviations}




\begin{tabular}{ll}
\hline AFM & Atomic Force Microscopy \\
\hline BSA-AF647 & Alexa Fluor 647 labelled variant of bovine serum albumin \\
\hline Cryo-EM & Cryo-electron microscopy \\
\hline DLS & Dynamic Light Scattering \\
\hline EPDELNs & Edible plant-derived exosome-like nanoparticles \\
\hline EVs & Extracellular vesicles \\
\hline F1 and F10 & First and tenth eluates in filtration procedure \\
\hline GF-EVs & Grapefruit-derived extracellular vesicles \\
\hline HSP70 & Human heat shock protein 70 \\
\hline HSP70-AF647 & Alexa Fluor 647 labelled variant of HSP70 \\
\hline NTA & Nanoparticle Tracking Analysis \\
\hline PBS & Phosphate-buffered saline \\
\hline PEVs & Plant-derived extracellular vesicles \\
\hline
\end{tabular}

\section{Declarations}

\section{Ethics approval and consent to participate}

Not applicable

\section{Consent for publication}

Not applicable

\section{Availability of data and materials}

Not applicable

\section{Competing interests}

The authors declare no conflict of interest.

\section{Funding}


The study was supported by the Russian Science Foundation project 19-74-20146 (cryo-EM, AFM, NTA, DLS experiments), the Russian Foundation for Basic Research project 18-29-09101 (sample preparation, flow cytometry, cell culture experiments). This work was partially supported by Ministry of Science and Higher Education of the Russian Federation (to A.K., Project \# 0657-2020-0004).

\section{Authors' contributions}

Conceptualization, T.S., A.K.; data curation, L.G., Y.G., S.L., E.V., V.B., R.K., E.K.; funding acquisition, T.S., I.G., A.K.; investigation, L.G., Y.G., S.L., E.V., V.B., R.K., E.K.; analysis and interpretation of data Y.K., A.M. B.M., A.L.K, I.A.V.; original draft preparation, T.S., Y.K.; L.G.; drafting the article or revising it critically for important intellectual content Y.K., A.K., A.L.K., I.A.V., I.G., B.M.; conception, design and, supervision T.S. All authors have read and agreed to the published version of the manuscript.

\section{Acknowledgments}

We thank Syeda Sadia Najam for a critical revision of the manuscript. The authors acknowledge the support and the use of resources of the Resource Center for Probe and Electron Microscopy at the NRC "Kurchatov Institute".

\section{References}

1. El Andaloussi S, Mäger I, Breakefield XO, Wood MJA. Extracellular vesicles: Biology and emerging therapeutic opportunities. Nat Rev Drug Discov [Internet]. Nature Publishing Group; 2013;12:347-57. Available from: http://dx.doi.org/10.1038/nrd3978

2. Van Niel G, D’Angelo G, Raposo G. Shedding light on the cell biology of extracellular vesicles. Nat Rev Mol Cell Biol [Internet]. Nature Publishing Group; 2018;19:213-28. Available from: http://dx.doi.org/10.1038/nrm.2017.125

3. Liao W, Du Y, Zhang C, Pan F, Yao Y, Zhang T, et al. Exosomes: The next generation of endogenous nanomaterials for advanced drug delivery and therapy. Acta Biomater. 2019;86:1-14.

4. Hessvik NP, Llorente A. Current knowledge on exosome biogenesis and release. Cell Mol Life Sci. Springer International Publishing; 2018;75:193-208.

5. Sil S, Dagur RS, Liao K, Peeples ES, Hu G, Periyasamy P, et al. Strategies for the use of Extracellular Vesicles for the Delivery of Therapeutics. J Neuroimmune Pharmacol. Journal of Neuroimmune Pharmacology; 2019;

6. Rashed MH, Bayraktar E, Helal GK, Abd-Ellah MF, Amero P, Chavez-Reyes A, et al. Exosomes: From garbage bins to promising therapeutic targets. Int J Mol Sci. 2017;18.

7. Shtam TA, Kovalev RA, Varfolomeeva EY, Makarov EM, Kil Y V., Filatov M V. Exosomes are natural carriers of exogenous siRNA to human cells in vitro. Cell Commun Signal [Internet]. 2013;11:88. Available from: https://doi.org/10.1186/1478-811X-11-88 
8. Li L, Lu S, Liang X, Cao B, Wang S, Jiang J, et al. үסTDEs: An Efficient Delivery System for miR-138 with Anti-tumoral and Immunostimulatory Roles on Oral Squamous Cell Carcinoma. Mol Ther Nucleic Acids [Internet]. Elsevier Ltd.; 2019;14:101-13. Available from: https://doi.org/10.1016/j.omtn.2018.11.009

9. Haney MJ, Klyachko NL, Zhao Y, Gupta R, Plotnikova EG, He Z, et al. Exosomes as drug delivery vehicles for Parkinson's disease therapy. J Control Release [Internet]. 2015/03/31. 2015;207:18-30. Available from: https://pubmed.ncbi.nlm.nih.gov/25836593

10. Tian Y, Li S, Song J, Ji T, Zhu M, Anderson GJ, et al. A doxorubicin delivery platform using engineered natural membrane vesicle exosomes for targeted tumor therapy. Biomaterials [Internet]. Elsevier Ltd; 2014;35:2383-90. Available from: http://dx.doi.org/10.1016/j.biomaterials.2013.11.083

11. Chen R, Huang H, Liu H, Xi J, Ning J, Zeng W, et al. Friend or Foe? Evidence Indicates Endogenous Exosomes Can Deliver Functional gRNA and Cas9 Protein. Small. 2019;15:1-13.

12. Meng W, He C, Hao Y, Wang L, Li L, Zhu G. Prospects and challenges of extracellular vesicle-based drug delivery system: considering cell source. Drug Deliv [Internet]. Taylor \& Francis; 2020;27:585-98. Available from: https://doi.org/10.1080/10717544.2020.1748758

13. Zhuang X, Deng Z Bin, Mu J, Zhang L, Yan J, Miller D, et al. Ginger-derived nanoparticles protect against alcohol-induced liver damage. J Extracell Vesicles. 2015;4:1-18.

14. Raimondo S, Naselli F, Fontana S, Monteleone F, Lo Dico A, Saieva L, et al. Citrus limon-derived nanovesicles inhibit cancer cell proliferation and suppress CML xenograft growth by inducing TRAILmediated cell death. Oncotarget. 2015;6:19514-27.

15. Xiao J, Feng S, Wang X, Long K, Luo Y, Wang Y, et al. Identification of exosome-like nanoparticlederived microRNAs from 11 edible fruits and vegetables. PeerJ. 2018;2018.

16. Lukasik A, Zielenkiewicz P. Plant MicroRNAs-novel players in natural medicine? Int J Mol Sci. 2017;18.

17. Cui Y, Gao J, He Y, Jiang L. Plant extracellular vesicles. Protoplasma. Protoplasma; 2020;257:3-12.

18. Wang Q, Zhuang X, Mu J, Deng Z Bin, Jiang H, Xiang X, et al. Delivery of therapeutic agents by nanoparticles made of grapefruit-derived lipids. Nat Commun [Internet]. Nature Publishing Group; 2013;4:1811-67. Available from: http://dx.doi.org/10.1038/ncomms2886

19. Wang B, Zhuang X, Deng Z Bin, Jiang H, Mu J, Wang Q, et al. Targeted drug delivery to intestinal macrophages by bioactive nanovesicles released from grapefruit. Mol Ther [Internet]. The American Society of Gene \& Cell Therapy; 2014;22:522-34. Available from: http://dx.doi.org/10.1038/mt.2013.190

20. Lazarev VF, Sverchinsky DV., Mikhaylova ER, Semenyuk PI, Komarova EY, Niskanen SA, et al. Sensitizing tumor cells to conventional drugs: HSP70 chaperone inhibitors, their selection and application in cancer models. Cell Death Dis [Internet]. Springer US; 2018;9. Available from: http://dx.doi.org/10.1038/s41419-017-0160-y

21. Landa SB, Korabliov PV, Semenova EV, Filatov MV. Peculiarities of the formation and subsequent removal of the circulating immune complexes from the bloodstream during the process of digestion. 
F1000Research [Internet]. F1000 Research Limited; 2018;7:618. Available from:

https://pubmed.ncbi.nlm.nih.gov/30079242

22. Nečas D, Klapetek P. Gwyddion: An open-source software for SPM data analysis. Cent Eur J Phys. 2012;10:181-8.

23. Emelyanov A, Shtam T, Kamyshinsky R, Garaeva L, Verlov N, Miliukhina I, et al. Cryo-electron microscopy of extracellular vesicles from cerebrospinal fluid. PLoS One. 2020;15:1-11.

24. Naryzhny SN. Blue Dry Western: Simple, economic, informative, and fast way of immunodetection. Anal Biochem. 2009;392:90-5.

25. Komarova EY, Afanasyeva EA, Bulatova MM, Cheetham ME, Margulis BA, Guzhova I V. Downstream caspases are novel targets for the antiapoptotic activity of the molecular chaperone Hsp70. Cell Stress Chaperones. 2004;9:265-75.

26. Sverchinsky DV., Nikotina AD, Komarova EY, Mikhaylova ER, Aksenov ND, Lazarev VF, et al. Etoposideinduced apoptosis in cancer cells can be reinforced by an uncoupled link between $\mathrm{Hsp70}$ and Caspase-3. Int J Mol Sci. 2018;19:1-15.

27. Vader P, Mol EA, Pasterkamp G, Schiffelers RM. Extracellular vesicles for drug delivery. Adv Drug Deliv Rev [Internet]. Elsevier B.V.; 2016;106:148-56. Available from:

http://dx.doi.org/10.1016/j.addr.2016.02.006

28. Chinnappan M, Srivastava A, Amreddy N, Razaq M, Pareek V, Ahmed R, et al. Exosomes as drug delivery vehicle and contributor of resistance to anticancer drugs. Cancer Lett [Internet]. Elsevier B.V.; 2020;486:18-28. Available from: https://doi.org/10.1016/j.canlet.2020.05.004

29. Luan X, Sansanaphongpricha K, Myers I, Chen H, Yuan H, Sun D. Engineering exosomes as refined biological nanoplatforms for drug delivery. Acta Pharmacol Sin [Internet]. Nature Publishing Group; 2017;38:754-63. Available from: http://dx.doi.org/10.1038/aps.2017.12

30. Akuma P, Okagu OD, Udenigwe CC. Naturally Occurring Exosome Vesicles as Potential Delivery Vehicle for Bioactive Compounds. Front Sustain Food Syst. 2019;3:1-8.

31. Rutter BD, Innes RW. Extracellular vesicles as key mediators of plant-microbe interactions. Curr Opin Plant Biol [Internet]. Elsevier Ltd; 2018;44:16-22. Available from: https://doi.org/10.1016/j.pbi.2018.01.008

32. Rybak K, Robatzek S. Functions of extracellular vesicles in immunity and virulence. Plant Physiol. 2019;179:1236-47.

33. Rutter BD, Innes RW. Extracellular vesicles isolated from the leaf apoplast carry stress-response proteins. Plant Physiol. 2017;173:728-41.

34. Pocsfalvi G, Turiák L, Ambrosone A, del Gaudio P, Puska G, Fiume I, et al. Protein biocargo of citrus fruit-derived vesicles reveals heterogeneous transport and extracellular vesicle populations. J Plant Physiol [Internet]. Elsevier; 2018;229:111-21. Available from:

https://doi.org/10.1016/j.jplph.2018.07.006

35. Chernyshev VS, Rachamadugu R, Tseng YH, Belnap DM, Jia Y, Branch KJ, et al. Size and shape characterization of hydrated and desiccated exosomes. Anal Bioanal Chem. 2015;407:3285-301. 
36. Issman L, Brenner B, Talmon Y, Aharon A. Cryogenic transmission electron microscopy nanostructural study of shed microparticles. PLoS One. 2013;8.

37. Linares R, Tan S, Gounou C, Arraud N, Brisson AR. High-speed centrifugation induces aggregation of extracellular vesicles. J Extracell Vesicles. 2015;4.

38. Yuana Y, Koning RI, Kuil ME, Rensen PCN, Koster AJ, Bertina RM, et al. Cryo-electron microscopy of extracellular vesicles in fresh plasma. J Extracell Vesicles. 2013;2:21494.

39. Naryzhny S, Volnitskiy A, Kopylov A, Zorina E, Kamyshinsky R, Bairamukov V, et al. Proteome of Glioblastoma-Derived Exosomes as a Source of Biomarkers. Biomedicines. Multidisciplinary Digital Publishing Institute; 2020;8:216.

40. Wang Q, Ren Y, Mu J, Egilmez NK, Zhuang X, Deng Z, et al. Grapefruit-derived nanovectors use an activated leukocyte trafficking pathway to deliver therapeutic agents to inflammatory tumor sites. Cancer Res. 2015;75:2520-9.

\section{Figures}

A

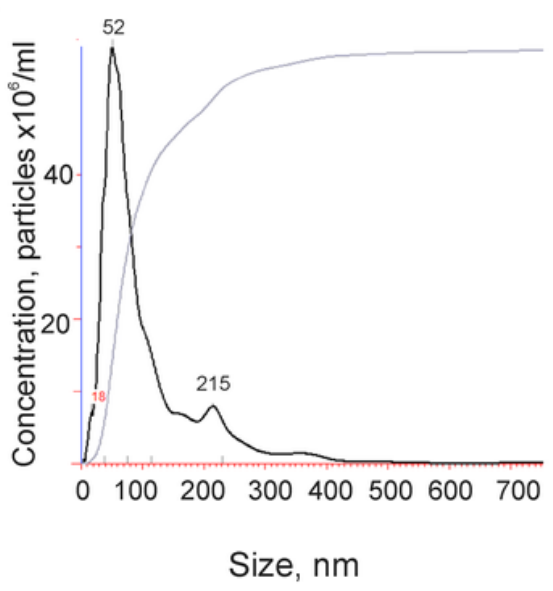

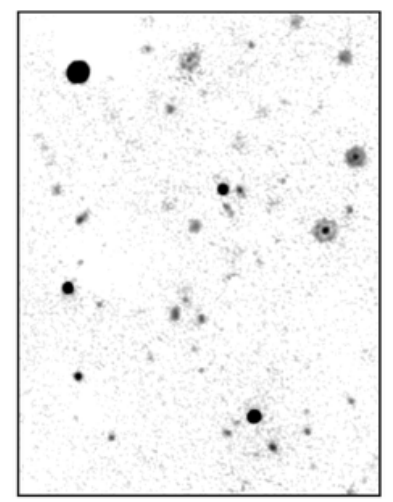

Sample video frame

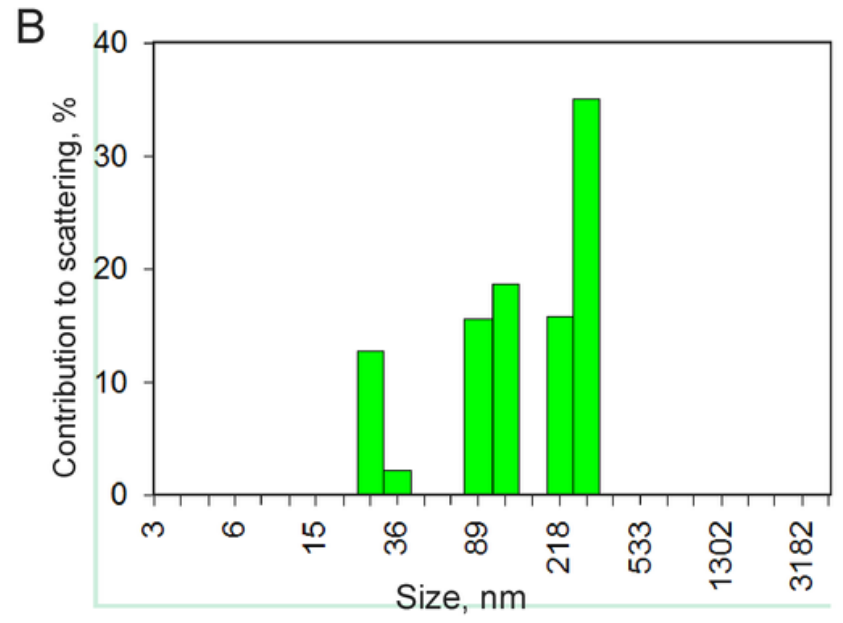

\section{Figure 1}

Characterization of grapefruit-derived particle size and concentration. a Nanoparticle tracking analysis (NTA) of nanovesicle size and concentration in the sample. b The size distribution of vesicles measured by dynamic light scattering (DLS). 
A
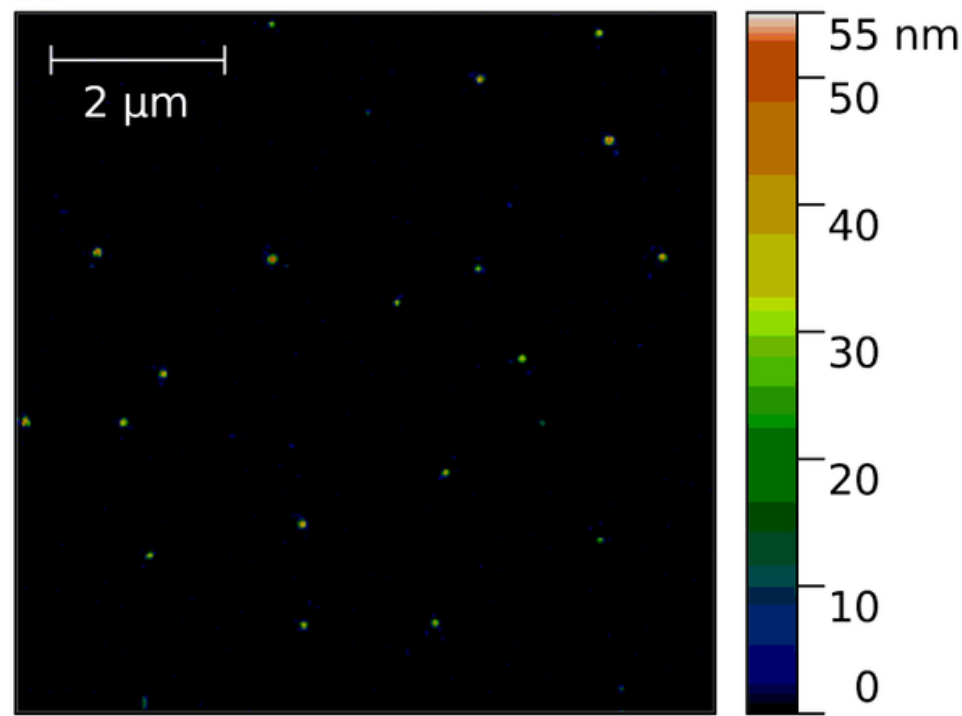

C
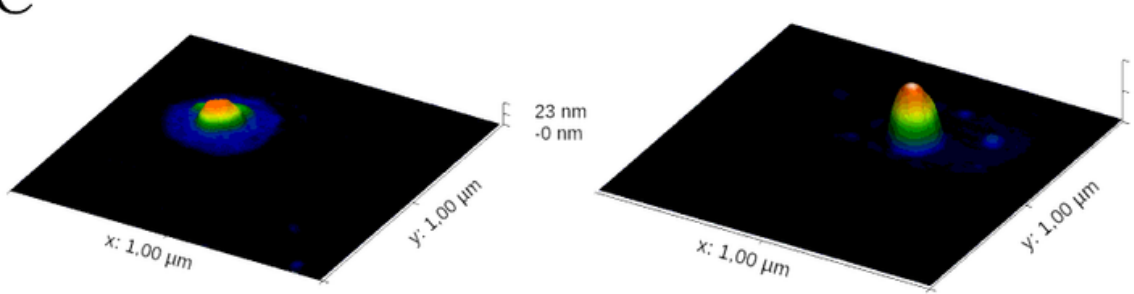

B

\section{Distribution of Object Heights}

0.9

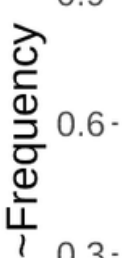

0.0
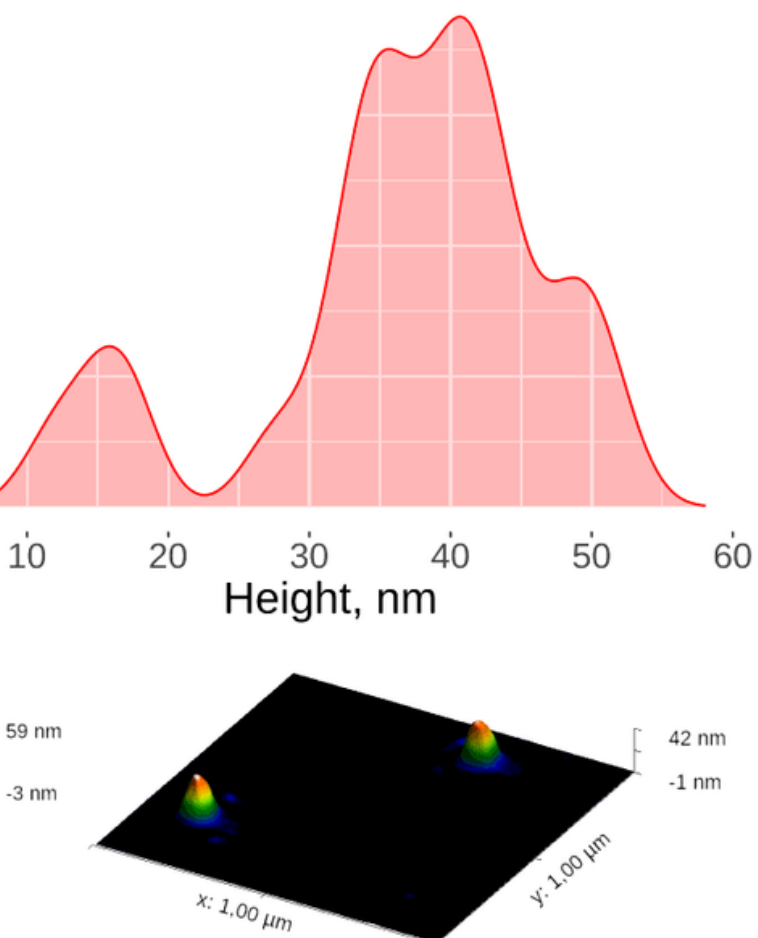

Figure 2

Characterization of grapefruit-derived nanoparticle size and morphology by Atomic Force Microscopy (AFM). a AFM images of grapefruit nanovesicles. b Height distribution of GF-EVs. c 3D images of some representative individual vesicles. 

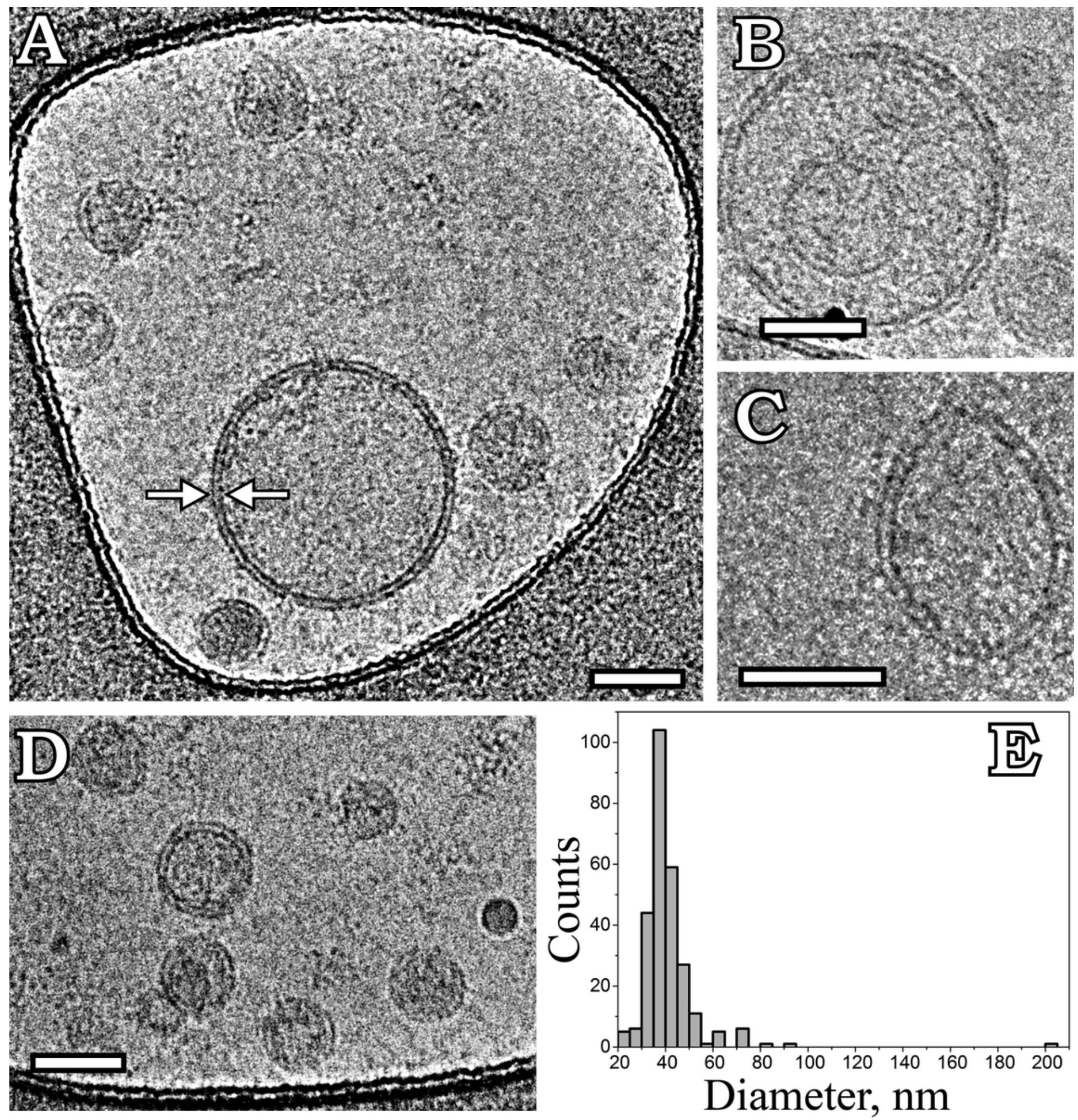

Figure 3

Cryo-EM images of grapefruit-derived extracellular vesicles (GF-EVs). a,b,d - round single vesicles; b double vesicle; c - elliptical vesicle; e - size distribution histogram. A total of 270 particles were analyzed. The arrows depict a lipid bilayer membrane of the vesicle. Scale bars are $50 \mathrm{~nm}$. 
A
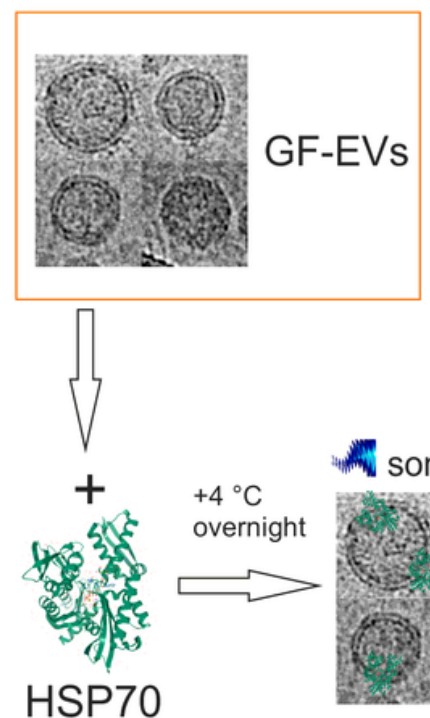

HSP70
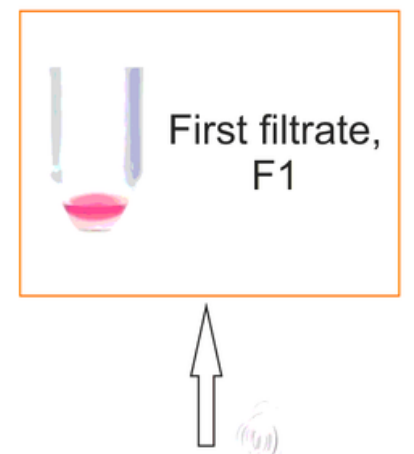

$100 \mathrm{kDa}$

Centricon

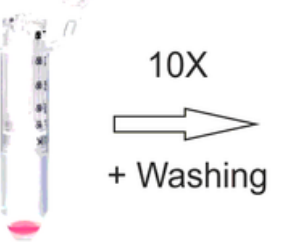

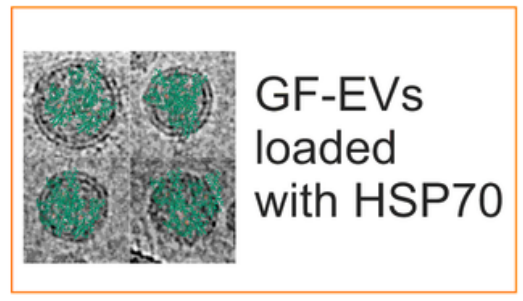

B
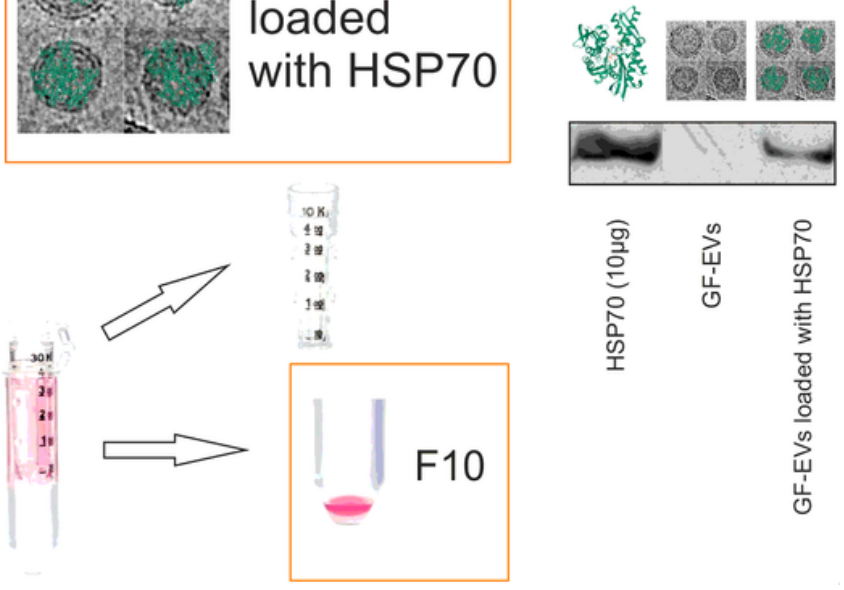

\section{Figure 4}

Loading efficiency of GF-EVs with HSP70 protein. a Flowchart summarizing the procedure of GF-EVs loading with HSP70. b Western blot of HSP70 in the initial and loaded samples of GF-EVs.

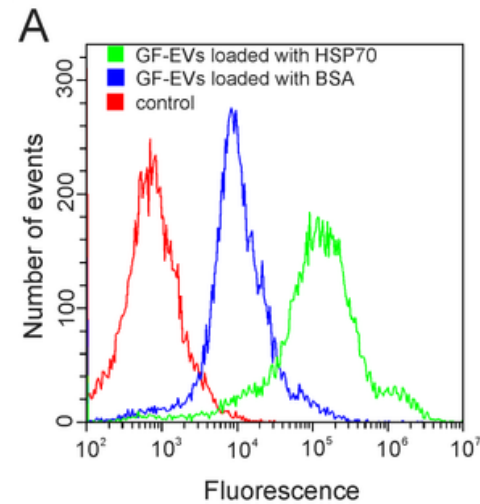

E

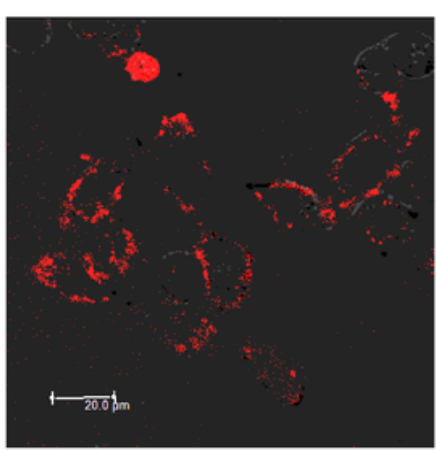

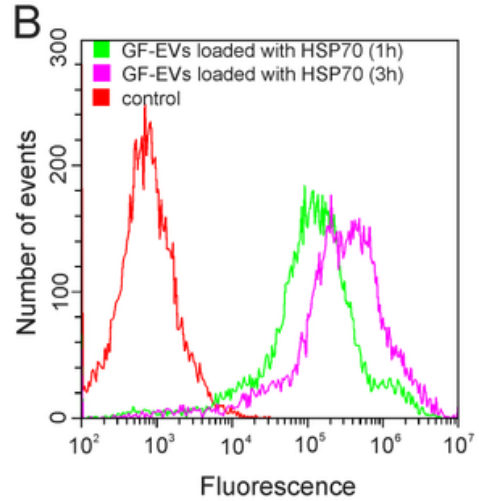

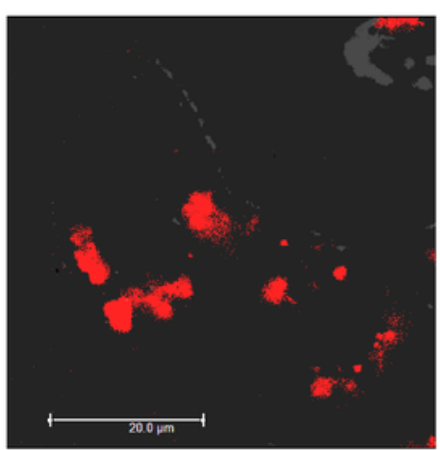

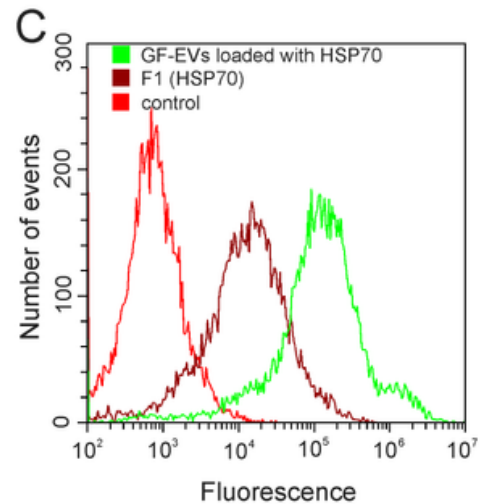

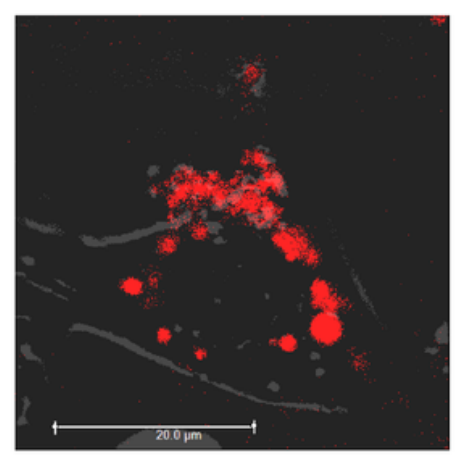

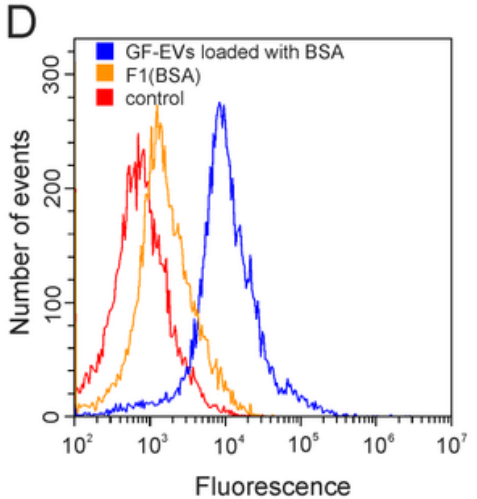

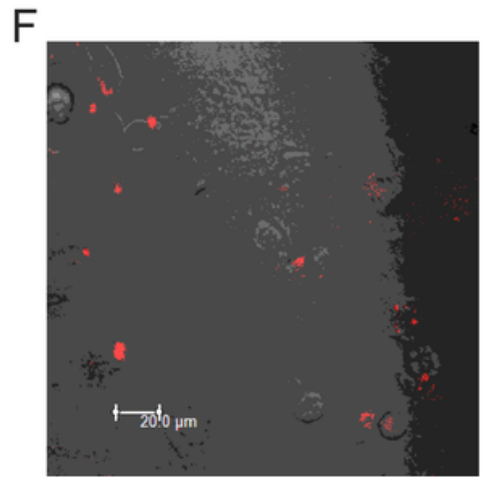

\section{G}

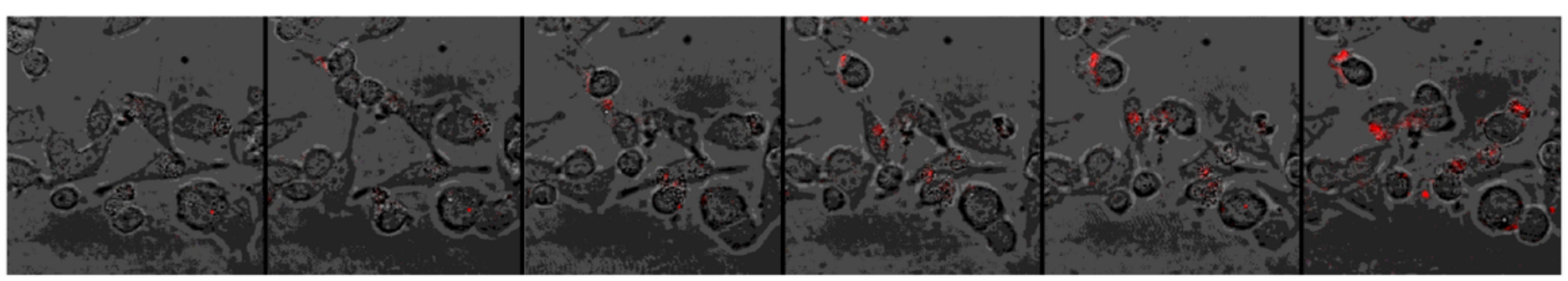


Figure 5

GF-EVs mediated delivery of exogenous proteins into the colorectal tissue culture cells analyzed by flow cytometry and confocal microscopy. a Incubation of HCT-116 cells with GF-EVs loaded with BSA-AF647 or HSP70-AF647 proteins for 1 hour. b Incubation of cells with GF-EVs loaded with HSP70-AF647 protein for 1 and 3 hours. c,d Incubation of cells with loaded GF-EVs in comparison with free HSP70-AF647 or BSA-AF647 proteins. e,f Micrographs of DLD1 cells co-cultivated with GF-EVs loaded with HSP70-AF647 (e) or BSA-AF647 (f) proteins. Scale bars are $20 \mu \mathrm{m}$. g The accumulation of fluorescent protein delivered to the recipient DLD1 cells by GF-EVs. Video recording frames detected every $30 \mathrm{~min}$ after adding of loaded GF-EVs to the cells.

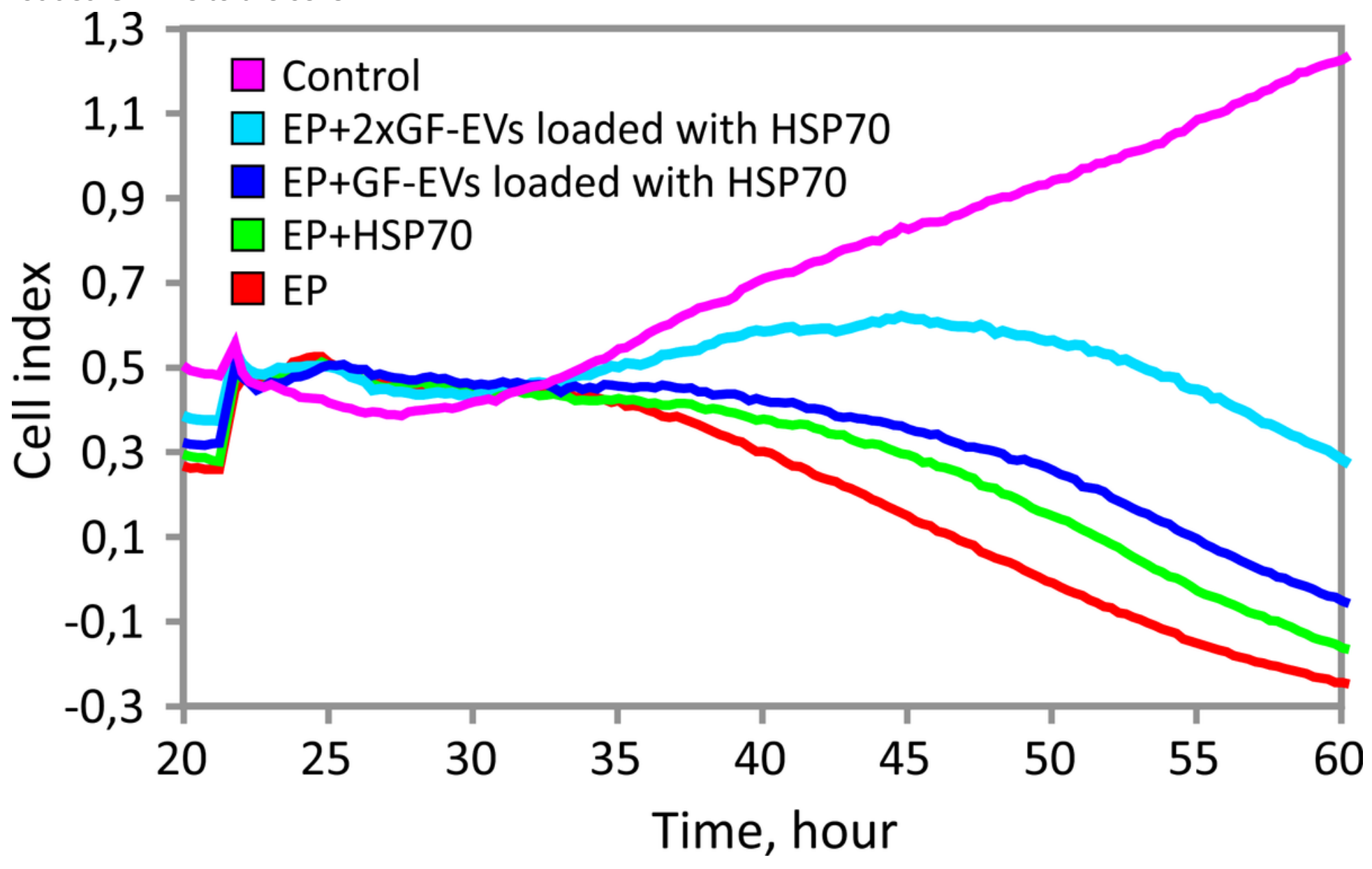

Figure 6

Protective effect of GF-EVs loaded with HSP70 to etoposide. DLD1-scr cells were seeded to wells of Eplates and after $16 \mathrm{~h}$ were incubated with HSP70 at concentration $50 \mu \mathrm{g} / \mathrm{mL}$ (green line) or GF-EVs loaded with HSP70 at concentrations 106 particles per cell (blue line) or 2x106 particles per cell (light blue line); $4 \mathrm{~h}$ later the etoposide (EP) to a final concentration of $20 \mu \mathrm{M}$ was added in each well except control cells (pink line). Recording with the aid of xCELLigence equipment was started immediately after drug added and lasted for $60 \mathrm{~h}$. Data from one of two independent experiments are presented.

\section{Supplementary Files}


This is a list of supplementary files associated with this preprint. Click to download.

- Supl.figure1.avi 